Pacific Journal of Mathematic 


\section{ON THE LEBESGUE AREA OF A DOUBLED MAP}

\section{Paul Slepian}

If $X$ is a metric space and $A$ is a non-empty closed subset of $X$ we construct a space $Y$ by doubling $X$ about $A$ in such a way that $X$ is imbedded homeomorphically in $Y$, the image of $A$ is the boundary of the image of $X$, and $X$ is also homeomorphic to the closure of the complement of its homeomorphic image in $Y$. In this way any function $f$ on $X$ may be doubled in a natural way to yield a function $F$ on $Y$. In 17 it is shown that if $X$ and $A$ satisfy certain triangulability conditions, and $f$ is continuous to Euclidean $n$ space, $E_{n}$, with $n \geqq k \geqq 2$, then $L_{k}(F) \leqq 2 L_{k}(f)$, with $L_{k}$ denoting $k$-dimensional Lebesgue area. In 18 , 21 and 22 the restrictions of 2-dimensionality are used to show that, when $k=2$, we have in fact $L_{2}(F)=2 L_{2}(f)$.

In particular if $(X, A)$ is a 2-dimensional manifold with boundary, then $Y$ is a compact 2-dimensional manifold. Furthermore, if $X$ is finitely triangulable, then $X$ and $A$ satisfy the required triangulability conditions and $L_{2}(F)=2 L_{2}(f)$. Thus to compute the Lebesgue area of $f$, we need only to know the Lebesgue area of $F$, whose domain is a compact 2-dimensional manifold.

Our terminology is consistent with [1]; however, some additional notations are cited below

\section{Notations.}

(i) 0 is the empty set,

(ii) $\{x\}$ is the set whose sole element is $x$.

(iii) $\sigma A=\{x \mid$ for some $y, x \in y \in A\}$.

(iv) $R$ is the set of real numbers.

(v) $A^{\cap}=\{x \mid x \subset A\}$.

(vi) $N(f, A, y)$ is the number of elements, possibly infinite, in the set $\{x \mid x \in A$ and $y=f(x)\}$.

(vii) $\operatorname{dmn} f=\{x \mid$ for some $y,(x, y) \in f\}$.

(viii) $\operatorname{rng} f=\{y \mid$ for some $x,(x, y) \in f\}$.

\section{AGREEMENT.}

(i) If $X$ is a topological space and $i$ is a positive integer, then $X^{i}=\{A \mid A$ is an $i$-cell in $A\}$.

Received May 27, 1958. This work was supported in part by a research grant from the National Science Foundation. This paper forms one chapter of a doctoral thesis presented at Brown University, June 1956. The author is indebted to Professor H. Federer for his supervision of this thesis, and his valuable suggestions and criticisms. 
(ii) If for some positive integer $i, A$ is an $i$-cell and $f$ is any homeomorphism of $A$ into $E_{i}$ note that the set $\{x \mid f(x) \in \operatorname{bdry} \operatorname{rng} f\}$

is independent of the homeomorphism $f$ selected. Consequently we agree to denote this unique set by $\hat{A}$.

3. Notations.

(i) If $n$ is a positive integer, $k$ is an integer and $k \leqq n$, then $H_{n}^{k}$ is $k$-dimensional Hausdorff measure over $E_{n}$.

(ii) Let $X$ be a $k$-dimensional finitely triangulable topological space and let $f$ be a continuous function on $X$ to $E_{n}$ with $k \leqq n$. Then $L_{k}(f)$ is the $k$-dimensional Lebesgue area of $f$. More precisely, $L_{k}(f)$ is the infimum of the set of all $t \in R$ such that for any $\varepsilon>0$ there exists a quasi-linear function $g$ on $X$ to $E_{n}$ such that $|g(x)-f(x)| \leqq \varepsilon$ for each $x \in X$ and $\int_{E_{n}} N(g, X, y) d H_{n}^{k} y<t$.

4. Definition. $X$ is a $k$-dimensional manifold if and only if $X$ is such a connected separable metric space that for any $x \in X$ there exists $A$ such that $A$ is an open $k$-cell in $X$ with $x \in A$.

5. Definition. $(X, A)$ is a 2-dimensional manifold with boundary if and only if the following conditions are satisfied:

(i) $X$ is a compact metric space and $A$ is a closed subset of $X$.

(ii) $X-A$ is a 2-dimensional manifold.

(iii) If $x \in A$, there exist $h$ and $\beta$ such that $\beta$ is an open subset of $X, x \in \beta$, and $h$ is a homeomorphism of $\beta$ onto $E_{2} \cap\left\{z \mid z_{2} \geqq 0\right\}$ such that $\operatorname{rng}(h \mid A)=E_{2} \cap\left\{z \mid z_{2}=0\right\}$.

Theorem. Let $(X, A)$ be a 2-dimensional manifold with boundary. Then

(i) $A$ has a finite number of components;

(ii) each component of $A$ is a simple closed curve.

Proof. $A$ is compact, and finitely many of the open sets $\beta$ described in 5 (iii) cover $A$. For each such $\beta$ the set $A \cap \beta$ is connected. Thus $A$ has a finite number of components.

Let $x$ be a component of $A$ and let $t \in x$. Then $\operatorname{ord}_{x} t=2,[2$, $\S 46]$, and $x$ is a simple closed curve. if :

7. Definition. $Y$ is obtained by doubling $X$ about $A$ if and only

(i) $X$ is a metric space and $A$ is a closed, non-empty subset of $A$; 
(ii) $Y$ is the topological space ( $\left.\mathrm{rng} g^{+} \cup \operatorname{rng} g^{-}\right) \subset(X \times R)$ where $g^{+}$and $g^{-}$are the functions on $X$ to $(X \times R)$ such that for each $x \in X$,

$$
\begin{aligned}
& g^{+}(x)=(x, \operatorname{dist}(\{x\}, A)), \\
& g^{-}(x)=(x,-\operatorname{dist}(\{x\}, A)) .
\end{aligned}
$$

8. Agrenment. Throughout this paper we fix $X, Y$ and $A$ such that $Y$ is obtained by doubling $X$ about $A$. In addition we agree to let $g^{+}$and $g^{-}$be the functions specified in 7 (ii).

9. Theorem. $g^{+}$and $g^{-}$are both homeomorphisms of $X$ into $Y$ such that

$$
\text { bdry } \operatorname{rng} g^{+}=\text {bdry rng } g^{-}=\operatorname{rng}\left(g^{+} \mid A\right)=\operatorname{rng}\left(g^{-} \mid A\right) .
$$

The proof is trivial.

10. THEOREM. If $(X, A)$ is a 2-dimensional manifold with boundary then $Y$ is a compact 2-dimensional manifold.

The proof is trivial.

11. Definition. The map $F$ is obtained by doubling the map $f$ if and only if $f$ is a function on $X$ and $F \circ g^{+}=F \circ g^{-}=f$.

12. Agreement. Throughout the remainder of this paper we fix and $F$ such that the map $F$ is obtained by doubling the map $f$.

\section{THEOREM.}

(i) $F$ is a function, $\operatorname{dmn} F=Y$, and $\operatorname{rng} F=\operatorname{rng} f$.

(ii) If $f$ is continuous, then $F$ is continuous.

(iii) If $X$ is compact and $f$ is light and continuous, then $F$ is light and continuous.

Proof. The proofs of (i) and (ii) are trivial.

Suppose $X$ is compact and let $z \in \operatorname{rng} F$. Then

$$
\{x \mid F(x)=z\}=\left(\operatorname{rng} g^{+} \cap\{x \mid F(x)=z\}\right) \cup\left(\operatorname{rng} g^{-} \cap\{x \mid F(x)=z\}\right) .
$$

Both sets on the right are closed in $Y$ and homeomorphic to $\{x \mid f(x)=z\}$ which is 0 -dimensional. Thus $\{x \mid F(x)=z\}$ is 0 -dimensional.

14. Definition. $(P, Q)$ is a finitely triangulable pair if and only if $P$ is a topological space, $Q \subset P$, and there exist $(K, \tau)$ and $K^{\prime}$ such that $(K, \tau)$ is a finite triangulation of $P, K^{\prime} \subset K$ and $\operatorname{rng}(\tau \mid Q)=\sigma K^{\prime}$. 
15. THEOREM. Let $(P, Q)$ be a 2-dimensional manifold with boundary, such that $P$ is finitely triangulable. Then $(P, Q)$ is a finitely triangulable pair.

The theorem is an immediate consequence of 6 .

16. Lemma. Let $X$ be k-dimensional and suppose that $(X, A)$ is a finitely triangulable pair. Let $\operatorname{rng} f \subset E_{n}$ with $n \geqq k$ and let $f$ be continuous. Let $\varepsilon>0$.

Let $(K, \tau)$ be a finite triangulation of $X$ in $E_{q}$ and let $K^{\prime} \subset K$ such that $\operatorname{rng}(\tau \mid A)=\sigma K^{\prime}$. Let $u$ be a quasi-linear function on $X$ to $E_{n}$ such that for each $B \in K,(u \circ$ inv $\tau) \mid B$ is a barycentric map of $B$, and such that for each $x \in X,|u(x)-f(x)| \leqq \varepsilon$.

Then there exists a quasi-linear function $h$ on $Y$ to $E_{n}$ such that $|h(y)-F(y)| \leqq \varepsilon$ for each $y \in Y$, and

$$
\int_{E_{n}} N(h, Y, y) d H_{n}^{k} y \leqq 2 \int_{E_{n}} N(u, X, y) d H_{n}^{k} y \text {. }
$$

Proof. We may suppose that $\tau$ is the identity map, $X \subset E_{q}$ and $Y \subset E_{q+1}$.

For each $B \in K$ let $B^{*}$ be the set of vertices of $B$. Then for each $B \in K$ let $\varphi_{B}^{+}$be the function which maps $B$ barycentrically onto the unique Euclidean simplex in $E_{q+1}$ spanned by the affinely independent set $\mathrm{rng}\left(g^{+} \mid B^{*}\right)$. More precisely, if $x \in B \in K$, and $\gamma_{x}$ is that unique function on $B^{*}$ to $R \cap\{y \mid 0 \leqq y \leqq 1\}$, such that $\sum_{t \in B^{*}} \gamma_{x}(t)=1$ and $x=\sum_{t \in B^{*}} \gamma_{x}(t) t$, then let $\varphi_{B}^{+}(x)=\sum_{t \in B^{*}} \gamma_{x}(t) g^{+}(t)$.

Similarly for each $B \in K$ let $\varphi_{\bar{B}}^{-}$be the function which maps $B$ barycentrically onto the unique Euclidean simplex in $E_{q+1}$ spanned by the set $\mathrm{rng}\left(g^{-} \mid B^{*}\right)$.

Then let

$$
H=\bigcup_{B \in K}\left(\left\{\operatorname{rng} \varphi_{B}^{+}\right\} \cup\left\{\operatorname{rng} \varphi_{B}^{-}\right\}\right) .
$$

Also let $\lambda^{+}$and $\lambda^{-}$be defined by,

$$
\lambda^{+}=\bigcup_{B \in K} \varphi_{B}^{+}, \quad \lambda^{-}=\bigcup_{B \in K} \varphi_{B}^{-} .
$$

Then let

$$
\eta=\left(\lambda^{+} \circ \operatorname{inv} g^{+}\right) \cup\left(\lambda^{-} \circ \operatorname{inv} g^{-}\right) .
$$

Since $(X, A)$ is a finitely triangulable pair, $\eta$ is a function and $(H, \eta)$ is a finite triangulation of $Y$ in $E_{q+1}$.

Next let

$$
h=\left(u \circ \operatorname{inv} g^{+}\right) \cup\left(u \circ \operatorname{inv} g^{-}\right) .
$$


Then $h$ is a function, and $(h \circ \operatorname{inv} \eta) \mid B^{\prime}$ is a barycentric map of $B^{\prime}$ for each $B^{\prime} \in H$. Thus $h$ is a quasi-linear map of $Y$ into $E_{n}$. Also $|h(y)-F(y)| \leqq \varepsilon$ for each $y \in Y$.

Finally,

$$
\begin{aligned}
& \int_{E_{n}} N(h, Y, y) d H_{n}^{k} y=\int_{E_{n}} N\left(h, \operatorname{rng} g^{+} \cup \operatorname{rng} g^{-}, y\right) d H_{n}^{k} y \\
& \qquad \int_{E_{n}} N\left(h, \operatorname{rng} g^{+}, y\right) d H_{n}^{k} y+\int_{E_{n}} N\left(h, \operatorname{rng} g^{-}, y\right) d H_{n}^{k} y \\
& \quad=2 \int_{E_{n}} N(u, X, y) d H_{n}^{k} y .
\end{aligned}
$$

17. Corollary. Let $X$ be k-dimensional and suppose that $(X, A)$ is a finitely triangulable pair. Let $\operatorname{rng} f \subset E_{n}$ with $n \geqq k$, and let $f$ bo continuous. Then $Y$ is finitely triangulable and $L_{k}(F) \leqq 2 L_{k}(f)$.

Proof. The construction of 16 guarantees that $Y$ is finitely triangulable. Now suppose that $2 L_{k}(f)<\delta<L_{k}(F)$. Let $\varepsilon>0$. It suffices to establish a quasi-linear function $h$ on $Y$ to $E_{n}$ such that $|h(y)-F(y)| \leqq \varepsilon$ for each $y \in Y$ and $\int_{E_{n}} N(h, Y, y) d H_{n}^{k} y<\delta$.

Let $(K, \tau)$ be a finite triangulation of $X$ in $E_{q}$ and let $V \subset K$ such that $\operatorname{rng}(\tau \mid A)=\sigma V$. By 6.24 of [1] there exists $K^{\prime}$ such that $K^{\prime}$ is a finite simplicial subdivision of $K$, and there exists a quasi-linear function $u$ on $X$ to $E_{n}$ such that $|u(x)-f(x)| \leqq \varepsilon$ for each $x \equiv X, \int_{E_{n}} N(u, X, y) d H_{n}^{k} y<\delta / 2$, and $(u \circ \operatorname{inv} \tau) \mid B^{\prime}$ is a barycentric map of $B^{\prime}$ for each $B^{\prime} \in K^{\prime}$.

Now let $V^{\prime}=K^{\prime} \cap \bigcup_{B \in V} B^{n}$. Clearly $\left(K^{\prime}, \tau\right)$ is a finite triangulation of $X$ in $E_{q}$, and since $K^{\prime}$ is a subdivision of $K$, we can state that rng $(\tau \mid A)=\sigma V^{\prime}$. Thus Lemma 16 applies to produce a quasi-linear function $h$ on $Y$ to $E_{n}$ such that $|h(y)-F(y)| \leqq \varepsilon$ for each $y \in Y$, and

$$
\int_{E_{n}} N(h, Y, y) d H_{n}^{k} y \leqq 2 \int_{E_{n}} N(u, X, y) d H_{n}^{k} y<\delta .
$$

18. Lemma. Let $B$ be a 2-cell metrized by $\rho$ and let $V \in B^{1} \cap \hat{B}^{n}$. Let $M=(V-\hat{V})$ and let $\varepsilon>0$. Then there exists a function $u$ such that:

(i) $u$ is a homeomerphism of $B$ into $B$.

(ii) $u(x)=x$ for $x \in(\hat{B}-M)$.

(iii) $\operatorname{rng}(u / M) \subset(B-\hat{B})$.

(iv) $M \cap \mathrm{rng} u=0$.

(v) $\rho(x, u(x))<\varepsilon$ for each $x \in B$. 
Proof. We may suppose that $B \subset E_{2}$. In fact, letting

$$
\begin{aligned}
& a=(1 / 2,1) \in E_{2}, \\
& \beta=(-1 / 2,1) \in E_{2}, \\
& \gamma=(0,0) \in E_{2},
\end{aligned}
$$

we may assume that $B$ is the convex hull of the set $\{a, \beta, \gamma\} \subset E_{2}$. Furthermore we may suppose that

$$
V=\{a t+(1-t) \beta \mid t \in\{z \mid 0 \leqq z \leqq 1\}\} .
$$

Now let $v$ be the function on $B-\{\gamma\}$ such that for each $x \in(B-\{\gamma\})$ we have $v(x)=[\sigma(V \cap\{t x \mid t \in R\})]_{1}$.

Then let $w$ be the function on $R$ such that $w(x)=(\varepsilon / 4)-\varepsilon x^{2}$. For each $x \in R$.

Finally let $u$ be the function on $B$ such that

$$
\begin{aligned}
& u(x)=[1-w(v(x))] x, \text { if } x \in(B-\{\gamma\}), \\
& u(\gamma)=\gamma .
\end{aligned}
$$

It is easy to check that $u$ satisfies the required conditions.

19. REMARK. Let $(K, \tau)$ be a finite triangulation of a topological space $P$ and let $\varepsilon>0$. Then by barycentrically subdividing each element of $K$, we obtain $K^{\prime}$ such that $\left(K^{\prime}, \tau\right)$ is a finite triangulation of $P, K^{\prime}$ is a finite simplicial subdivision of $K$ and each element of $K^{\prime}$ is less than $\varepsilon$ in diameter.

20. Definition. A subset $V$ of $E_{n}$ is k-removable $([1,6.26])$ if and only if $V$ is a closed set with the following property.

If $u$ is a continuous function on a $k$-dimensional finitely triangulable space $T$, to $E_{n}$, and

$G=\{P \mid P$ is a finitely triangulable subset of $T$ and $\operatorname{rng}(u \mid P) \cap V=0\}$, then $L_{k}(u)=\sup _{P \in G} L_{k}(u \mid P)$.

In the following lemma we make use of the fact that any finite subset of $E_{n}$ is $k$ removable.

21. Lemma. Let $M$ be a metric space. Let $K$ be a finite 2-dimensional cell-complex in $M$ such that $M=\sigma K$. Suppose there exists a finite non-empty set $P \subset\left(K \cap M^{1}\right)$ and a function $r$ on $P$ such that for each $x \in P$

(i) $\{\gamma(x)\}=\left\{B \mid\left(B \in\left(K \cap M^{2}\right)\right)\right.$ and $\left.(x \subset \hat{B})\right\}$, and $^{1}$

1 Geometrically the conditions (i) and (ii) state that each 1 -cell of $P$ is a subset of the boundary of exactly one 2-cell of $K$, and futhermore, this 2-cell of $K$ meets no other element of $P$. 
(ii) $\sigma P \cap \gamma(x)=x$.

Let $J$ be that set of all $M^{\prime}$ such that $M^{\prime}$ is a finitely triangulable subset of $M$ and $M^{\prime} \cap \sigma P=0$. Let $u$ be a continuous function on $M$ to $E_{n}$ with $n \geqq 2$. Then $L_{\mathrm{z}}(u)=\sup _{u \in J} L_{\mathrm{z}}\left(u \mid M^{\prime}\right)$.

Proof. Let $\rho$ metrize $M$. It suffices to show that $L_{2}(u) \leqq$ $\sup _{\mathbf{u}^{\prime} \in J} L_{2}\left(u \mid M^{\prime}\right)$.

The remainder of the proof is divided into 2 parts.

Part 1. Let $\varepsilon>0$. There exists a function $\varphi$ such that:

(i) $\varphi$ is a homeomorphism of $M$ into $M$.

(ii) For each $x \in M, \rho(x, \varphi(x))<\varepsilon$.

(iii) $\bigcup_{B \in P} \hat{B}=\sigma P \cap \operatorname{rng} \varphi=\sigma P \cap \operatorname{rng}(\varphi \mid \sigma P)$.

Proof of Part 1. For each $x \in P$ we apply Lemma 18 to produce a function $d_{x}$ is satisfying the following conditions:

( i') $d_{x}$ is a homeomorphism of $\gamma(x)$ into $\gamma(x)$.

(ii') $d_{x}(t)=t$, for $\mathrm{t} \in[\widehat{\gamma(x)}-(x-\hat{x})]$.

(iii') $\operatorname{rng}\left(d_{x} \mid(x \hat{-x})\right) \subset(\gamma(x)-\widehat{\gamma(x)})$.

(iv') $(x-\hat{x}) \cap \operatorname{rng} d_{x}=0$.

$\left(\mathrm{v}^{\prime}\right)$ For each $t \in \gamma(x), \rho\left(d_{x}(t), t\right)<\varepsilon$.

Let $\Psi$ be the identity map of $(M-\sigma \operatorname{rng} \gamma)$ onto itself and let

$$
\varphi=\bigcup_{x \in P} d_{x} \cup \Psi
$$

Part 2. $L_{2}(u) \leqq \sup _{M^{\prime} \in J} L_{2}\left(u \mid M^{\prime}\right)$.

Proof of Part 2. Let $\varepsilon>0$ and produce a function $\varphi$ satisfying the conditions (i)-(iii) of part 1 .

The finite set $\operatorname{rng}(u \mid[\sigma P \cap \operatorname{rng} \varphi])$ is 2-removable. Thus if we let $W$ be the set of all $Q$ such that $Q$ is a finitely triangulable subset of rng $\varphi$ and

$$
\operatorname{rng}(u \mid Q) \cap \operatorname{rng}(u \mid[\sigma P \cap \operatorname{rng} \varphi])=0
$$

we can state that

$$
L_{2}(u \circ \varphi)=L_{2}(u \mid \operatorname{rng} \varphi)=\sup _{Q \in W} L_{2}(u \mid Q) \leqq \sup _{M^{\prime} \in J} L_{L}\left(u \mid M^{\prime}\right) .
$$

Due to the arbitrary nature of $\varepsilon$ we have

$$
L_{2}(u) \leqq L_{2}(u \circ \varphi) \leqq \sup _{M^{\prime} \in J} L_{2}\left(u \mid M^{\prime}\right) .
$$

22. Corollary. Let $K$ be a finite 2-dimensional cell complex in $X$ such that $X=\sigma K$. Suppose there exists a finite non-empty set $P \subset\left(K \cap X^{1}\right)$ 
such that $A \subset \sigma P$, and there exists a function $\gamma$ on $P$ such that for each $x \in P$,

and

$$
\{\gamma(x)\}=\left\{B \mid\left(B \in\left(K \cap X^{2}\right)\right) \text { and }(x \subset \hat{B})\right\},
$$

$$
\sigma P \cap \gamma(x)=x .
$$

Let $\operatorname{rng} f \subset E_{n}$ with $n \geqq 2$ and let $f$ be continuous. Then $2 L_{2}(f) \leqq L_{2}(F)$.

Proof. Let $J$ be the set of all $X^{\prime}$ such that $X^{\prime}$ is a finitely triangulable subset of $X$ and $X^{\prime} \cap \sigma P=0$. Let $V \in J$. Then since

$$
\operatorname{rng}\left(g^{+} \mid V\right) \cap \operatorname{rng}\left(g^{-} \mid V\right)=0 \text {, }
$$

we infer that,

$$
\begin{gathered}
L_{2}\left(F \mid \operatorname{rng}\left(g^{+} \mid V\right)\right)+L_{2}\left(F \mid \operatorname{rng}\left(g^{-} \mid V\right)\right)=L_{2}\left(F \mid\left(\operatorname{rng}\left(g^{+} \mid V\right) \cup \operatorname{rng}\left(g^{-} \mid V\right)\right)\right) \\
\quad \text { Since } F \circ g^{-}=F \circ g^{-}=f, \\
\quad L_{2}(F) .
\end{gathered}
$$

$$
2 L_{2}(f \mid V) \leqq L_{2}(F),
$$

and

$$
2 L_{2}(f)=2 \sup _{X^{\prime} \in J} L_{2}\left(f \mid X^{\prime}\right) \leqq L_{2}(F) .
$$

23. Corollary. Suppose that $(X, A)$ is a 2-dimensional manifold with boundary and $X$ is finitely triangulable. Let $\operatorname{rng} f \subset E_{n}$ with $n \geqq 2$ and let $f$ be continuous. Then $2 L_{2}(f)=L_{2}(F)$.

Proof. From 15 and 17 we infer that $L_{2}(F) \leqq 2 L_{2}(f)$.

Let $(K, \tau)$ be a finite triangulation of $X$. By appropriately subdividing each 2-cell of $K$ we can easily produce $H$ such that $H$ is a finite 2-dimensional cell-complex in $X, \sigma H=X$, and such that $B \cap A \in H$ for each $B \in H$ with $B \cap A \neq 0$. Let $P=A^{\cap} \cap H \cap X^{1}$. Note that if $x \in P$, then the set

$$
\left\{B \mid\left(B \in\left(H \cap X^{2}\right)\right) \text { and }(x \subset \hat{B})\right\}
$$

has precisely one element.

Thus let $\gamma$ be the function on $P$ such that for each $x \in P$

$$
\gamma(x)=\sigma\left\{B \mid\left(B \in\left(H \cap X^{2}\right)\right) \text { and }(x \subset \hat{B})\right\} .
$$

The construction of $H$ guarantees that $\sigma P \cap \gamma(x)=x$ for each $x \in P$. Thus 22 applies and $2 L_{2}(f)=L_{2}(F)$.

\section{REFERNCES}

1. H. Federer, On Lebesgue area, Ann. of Math. 61, (1955), 289.

2. C. Kuratowski, Topolojie, Monografie Matematyczne, 21, Warsaw (1950).

HUGHES RESEARCH LABORATORIES 


\section{PACIFIC JOURNAL OF MATHEMATICS}

\section{EDITORS}

David Gilbarg

Stanford University

Stanford, California

R. A. Beaumont

University of Washington

Seattle 5, Washington

\author{
A. L. Whiteman
}

University of Southern California

Los Angeles 7, California

E. G. Straus

University of California

Los Angeles 24, California

\section{ASSOCIATE EDITORS}

\author{
E. F. BECKENBACH \\ C. E. BURGESS \\ M. HALL \\ E. HEWITT
}

\author{
A. HORN \\ V. GANAPATHY IYER \\ R. D. JAMES \\ M. S. KNEBELMAN
}

L. NACHBIN
I. NIVEN
T. G. OSTROM
H. L. ROYDEN

M. M. SCHIFFER

G. SZEKERES

F. WOLF

K. YOSIDA

\section{SUPPORTING INSTITUTIONS}

\author{
UNIVERSITY OF BRITISH COLUMBIA \\ CALIFORNIA INSTITUTE OF TECHNOLOGY \\ UNIVERSITY OF CALIFORNIA \\ MONTANA STATE UNIVERSITY \\ UNIVERSITY OF NEVADA \\ OREGON STATE COLLEGE \\ UNIVERSITY OF OREGON \\ UNIVERSITY OF SOUTHERN CALIFORNIA
}

\author{
STANFORD UNIVERSITY \\ UNIVERSITY OF UTAH \\ WASHINGTON STATE COLLEGE \\ UNIVERSITY OF WASHINGTON \\ AMERICAN MATHEMATICAL SOCIETY \\ CALIFORNIA RESEARCH CORPORATION \\ HUGHES AIRCRAFT COMPANY \\ THE RAMO-WOOLDRIDGE CORPORATION
}

Mathematical papers intended for publication in the Pacific Journal of Mathematics should be typewritten (double spaced), and the author should keep a complete copy. Manuscripts may be sent to any of the editors. All other communications to the editors should be addressed to the managing editor, E. G. Straus at the University of California, Los Angeles 24, California.

50 reprints per author of each article are furnished free of charge; additional copies may be obtained at cost in multiples of 50 .

The Pacific Journal of Mathematics is published quarterly, in March, June, September, and December. The price per volume (4 numbers) is $\$ 12.00$; single issues, $\$ 3.50$. Back numbers are available. Special price to individual faculty members of supporting institutions and to individual members of the American Mathematical Society: $\$ 4.00$ per volume; single issues, $\$ 1.25$.

Subscriptions, orders for back numbers, and changes of address should be sent to Pacific Journal of Mathematics, 2120 Oxford Street, Berkeley 4, California.

Printed at Kokusai Bunken Insatsusha (International Academic Printing Co., Ltd.), No. 6, 2-chome, Fujimi-cho, Chiyoda-ku, Tokyo, Japan.

\section{PUBLISHED BY PACIFIC JOURNAL OF MATHEMATICS, A NON-PROFIT CORPORATION}

The Supporting Institutions listed above contribute to the cost of publication of this Journal, but they are not owners or publishers and have no responsibility for its content or policies. 


\section{Pacific Journal of Mathematics}

\section{Vol. 8, No. 3 \\ May, 1958}

Michael Israel Aissen, A set function defined for convex plane domaines... . 383

Robert Ellis, Distal transformation groups ................... 401

Ciprian Foias, On a commutative extension of a commutative Banach algebra ....................................... 407

Jerry William Gaddum, Linear inequalities and quadratic forms ......... 411

Allen A. Goldstein and Elliott Ward Cheney, Jr., A finite algorithm for the solution of consistent linear equations and inequalities and for the Tchebycheff approximation of inconsistent linear equations...........

William L. Hart and T. S. Motzkin, Proof of the fundamental theorem on implicit functions by use of composite gradient corrections .......... 429

Henry Berge Helson, Conjugate series and a theorem of Paley .......... 437

Wu-Chung Hsiang, Abelian groups characterized by their independent subsets....................................... 447

John W. Lamperti, On the isometries of certain function-spaces ........ 459

Karel DeLeeuw and Walter Rudin, Extreme points and extremum problems

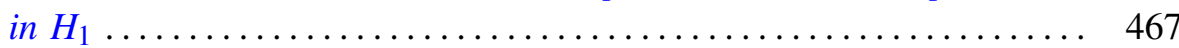

Eugene Lukacs, Some extensions of a theorem of Marcinkiewicz........ 487

George W. Mackey, Multiplicity free representations of finite groups ..... 503

Eben Matlis, Injective modules over Noetherian rings ............. 511

John William Neuberger, Continuous products and nonlinear integral equations

Lawrence Edward Payne and Hans F. Weinberger, New bounds for solutions of second order elliptic partial differential equations...

William T. Reid, A Prüfer transformation for differential systems ........ 575

Howard L. Rolf, The free lattice generated by a set of chains ...

K. M. Saksena, Inversion and representation theorems for a generalized

Laplace integral....................................... 597

Daniel Shanks, Two theorems of Gauss......................... 609

Paul Slepian, On the Lebesgue area of a doubled map ............... 613

Otto Szász and Nelson Paul Yeardley, Jr., The representation of an analytic function by general Laguerre series . ..................... 621

Alan C. Woods, On two-dimensional convex bodies ................. 635 